\title{
ENGORDE DE CONEJOS ALIMENTADOS CON Tithonia diversifolia, Trichanthera gigantea y Arachis pintoi
}

\section{FATTENING OF RABBIT FEEDED WITH Tithonia diversifolia, Trichanthera gigantea AND Arachis pintoi}

\begin{abstract}
Gastón Castaño ${ }^{1}$, Jaime Cardona ${ }^{2}$
${ }^{1}$ Zootecnista, M.Sc. Profesor Asociado, Facultad de Ciencias Pecuarias. Corporación Universitaria Santa Rosa de Cabal, Grupo de Investigación en Nutrición y Alimentación Animal. Campus El Jazmín km 4 vía Chinchiná, Santa Rosa de Cabal, Risaralda, Colombia, e-mail: gaston.castano@unisarc.edu.co; ${ }^{2}$ Zootecnista, Especialista en Gestión y Producción Piscícola. Profesor Asistente, Facultad de Ciencias Pecuarias. Corporación Universitaria Santa Rosa de Cabal, Grupo de Investigación en Nutrición y Alimentación Animal. Campus El Jazmín km 4 vía Chinchiná, Santa Rosa de Cabal, Risaralda, Colombia, email: jaime.cardona@unisarc.edu.co
\end{abstract}

Rev. U.D.C.A Act. \& Div. Cient. 18(1): 147-154, Enero-Junio, 2015

\section{RESUMEN}

En el trópico existen recursos forrajeros que poseen un gran potencial en la alimentación de conejos y, además, pueden ser producidos en grandes cantidades. El objetivo de este trabajo fue evaluar el efecto de suministrar forraje fresco de Tithonia diversifolia, Trichanthera gigantea y Arachis pintoi en la alimentación de conejos, durante la fase de ceba, sobre el desempeño productivo. Se utilizaron 16 conejos de la raza Nueva Zelanda Blanco, 8 hembras y 8 machos, con 30 días de edad y peso vivo de $976 \pm 63 \mathrm{~g}$, que fueron distribuidos en los tratamientos, según un diseño experimental bloques al azar, utilizando el sexo del animal, como factor de bloqueo. El experimento presentó una duración de 35 días. Los tratamientos consistieron en cuatro dietas suministradas a conejos, durante la fase de engorde, que se diferenciaron según el tipo de forraje utilizado: un control en donde sólo se suministró concentrado a voluntad y, otros tres, en los que, adicionalmente, se proporcionaron $180 \mathrm{~g} \mathrm{FV/día} \mathrm{de} T$. diversifolia, T. gigantea o A. pintoi. Los animales fueron sacrificados a los 65 días de edad. El suministro de forraje no afectó el peso ni el consumo de concentrado. El consumo total de alimento, concentrado+forraje, fue mayor para los conejos que recibieron el $A$. pintoi frente a los que recibieron T. gigantea, pero no en comparación con $T$. diversifolia.

Palabras clave: Conejos raza Nueva Zelanda Blanco, botón de oro, quiebrabarrigo, maní forrajero, forrajes tropicales.

\section{SUMMARY}

There are forage resources in the tropic with great potential in diets for rabbits, which that can be produced in large quantities. The aim of this research was to evaluate the effect on the performance of using in the diet Tithonia diversifolia, Trichanthera gigantea and Arachis pintoi as fresh fodder in the diet of fattening rabbits. Sixteen rabbits of the White New Zealand breed, eight females and eight males, with 30 days of age and live weight of $976 \pm 63 \mathrm{~g}$ were used, in a randomized block design, using the sex of the animal as the blocking factor. The experiment lasted 35 days. Treatments consisted of four diets fed to rabbits during the fattening phase, differentiated according to the forage type used: a control where rabbits were only supplied with concentrate ad libitum and three treatments in which additionally $180 \mathrm{~g} /$ day of either $T$. gigantea, $T$. diversifolia or A. pintoi were offered. The animals were slaughtered at 65 days of age. Forage supply did not affect neither the weight nor the consumption of concentrate. The total consumption of feed, concentrate+forage, was higher in rabbits receiving A. pintoi compared with those who received $T$. gigantea, but not compared to T. diversifolia.

Key words: White New Zealand breed rabbits, Mexican sunflower, yatago, pinto peanut, tropical forages.

\section{INTRODUCCIÓN}

Las particularidades del sistema digestivo de los conejos permite la utilización de alimentos que, para otras especies no rumiantes, generan baja productividad, pues esta especie puede lograr una provechosa utilización de fuentes fibrosas en la dieta (Nieves et al. 2009b), debido a la fermentación en el ciego; además, el proceso de cecotrofia maximiza el aprovechamiento del alimento (Nieves et al. 2009a).

La alimentación representa cerca del $70 \%$ de los costos asociados a la producción de conejos para carne (Maertens, 
1999). Las materias primas convencionales son escasamente disponibles y costosas, de tal manera que es necesario desarrollar estrategias alimenticias para conejos, con base en recursos disponibles en la zona intertropical y, para lo cual, el uso de forrajes ha generado un creciente interés (Nieves et al. 2008; 2009b; 2011). En la franja intertropical existe un potencial para producir biomasa vegetal y se encuentran diversas especies que pueden tener valor alimenticio para animales herbívoros, lo que incentiva el estudio de estrategias de alimentación que consideren el manejo de forrajes (Nieves et al. 2008), lo cual, puede llegar a ser beneficioso para la producción de conejos, cuando se suministran acompañados de un suplemento concentrado (lyegue-Erakpotobor \& Muhammad, 2008). Se ha evaluado la digestibilidad de Tithonia diversifolia, de Trichanthera gigantea y de Arachis pintoi (Nieves et al. 2008; 2009a; 2011), en conejos y los resultados sugieren que estas fuentes forrajeras poseen un importante potencial en la alimentación de esta especie y es necesario evaluar la respuesta de productiva de los conejos, cuando dichos forrajes son incluidos en la dieta. Es escasa la información del efecto de suministrar estos forrajes frescos sobre el desempeño productivo, bajo condiciones locales.

El objetivo de este trabajo fue evaluar el efecto de suministrar forraje fresco de T. diversifolia, T. gigantea y A. pintoi sobre el desempeño productivo de conejos, que consumen concentrado, durante la fase de ceba.

\section{MATERIALES Y MÉTODOS}

Localización. El trabajo, se realizó en la granja pecuaria experimental "El Jazmín", localizada en el municipio de Santa
Rosa de Cabal, perteneciente al departamento de Risaralda, en Colombia, a los $4^{\circ} 52^{\prime} 07^{\prime \prime}$ ' LN y 75³7'22' ' LW y a $1701 \mathrm{msnm}$, con una temperatura media de $18,6^{\circ} \mathrm{C}$ y una precipitación anual de $2620 \mathrm{~mm}$.

Duración y tratamientos. El experimento presentó una duración de 35 días, seis de acostumbramiento a las dietas experimentales y 29 de recolección de información. Los tratamientos consistieron en cuatro dietas suministradas a conejos, durante la fase de engorde, que se diferenciaron según el tipo de forraje utilizado: un control en donde sólo se suministró concentrado a voluntad (C) y, otros tres, en los que, adicionalmente, se proporcionaron $180 \mathrm{~g}$ de FV/día, ya sea de T. gigantea (TG), T. diversifolia (TD) o A. pintoi (AP). Las características del concentrado y de los forrajes utilizados en el experimento se pueden apreciar en la tabla 1.

Animales y manejo. Se utilizaron 16 conejos de la raza Nueva Zelanda Blanco, 8 hembras y 8 machos, con 30 días de edad. El peso vivo promedio de las hembras fue $942 \pm 46 \mathrm{~g}$, el de los machos $1010 \pm 61 \mathrm{~g}$ y el de todos animales $976 \pm 63 \mathrm{~g}$. Los conejos fueron distribuidos aleatoriamente en los tratamientos, según un diseño experimental bloques al azar generalizado, utilizando el sexo del animal, como factor de bloqueo. Los animales, se alojaron individualmente en jaulas, dotadas de beberos manuales y de bandejas, para la recolección individual de heces y de desperdicio. Las dimensiones de las jaulas fueron $50 \mathrm{~cm}$ de ancho, $35 \mathrm{~cm}$ de alto y $50 \mathrm{~cm}$ largo. El forraje empleado, se cortó el día anterior al suministro; se pesó a las 7:00h y se repartió en dos dosis: la primera, a las 7:00h y, la segunda, a las 16:00h. Para garantizar el consumo a voluntad del concentrado, se suministró

Tabla 1. Composición química del concentrado y de los forrajes utilizados en el experimento.

\begin{tabular}{|l|c|c|c|c|}
\hline \multicolumn{1}{|c|}{ Fracción del alimento } & Concentrado & T. diversifolia & A. pintoi & T. gigantea \\
\hline Materia seca, g/kg & 910,0 & 218,1 & 402,3 & 229,4 \\
\hline Proteína cruda, g/kg de MS & 189,7 & 307,0 & 204,4 & 153,6 \\
\hline Extracto etéreo, g/kg de MS & 37,2 & 35,9 & 69,9 & 67,1 \\
\hline CNFF $^{1}$, g/kg de MS & 439,1 & 161,9 & 114,8 & 178,8 \\
\hline FDN, g/kg de MS & 255,0 & 327,8 & 523,4 & 389,6 \\
\hline FDA, g/kg de MS & 139,5 & 235,0 & 374,7 & 270,5 \\
\hline Lignina, g/kg de MS & 50,9 & 90,9 & 121,4 & 102,6 \\
\hline Cenizas, g/kg de MS & 79,0 & 167,4 & 87,5 & 210,9 \\
\hline Energía bruta, kcal/kg MS & 4341 & 4105 & 4063 & 3897 \\
\hline
\end{tabular}

${ }^{1}$ Carbohidratos no fibrosos $=100-$ FDN - PC - EE - cenizas. 
una cantidad equivalente al consumo del día anterior más el $5 \%$, que fue repartido en dos dosis: la primera, a las 7:00h y, la segunda, a las 13:00h.

Los animales fueron sacrificados el día 35 del experimento, cuanto tenían 65 días de edad.

Los animales, se pesaron los días 0, 7, 14, 21 y 35 de iniciado el experimento; el pesaje se realizó a las 7:00h y previo al suministro de alimento. Para determinar el consumo de alimento, se pesó el suministro diario de alimento y el desperdicio del día anterior, a las 7:00h, entre los días 7 y el 34 . Una vez se sacrificaron los animales, se ponderó la canal, la piel, la cabeza, las patas, el corazón, el hígado, el intestino y los pulmones.

Recolección de muestras. Todos los días se colectaron, aproximadamente, $180 \mathrm{~g}$ de cada forraje, se empacaron en bolsas plásticas, se almacenaron a $-20^{\circ} \mathrm{C}$, se deshidrataron en horno de aire forzado a $60^{\circ} \mathrm{C}$ por 48 horas. Las muestras deshidratadas entre los días 28 y 35 se molieron, a través de un molino $U d y \AA$, con criba de $1 \mathrm{~mm}$ y se mezclaron para formar una muestra compuesta, que fue enviada a laboratorio para su análisis químico. El día 32, se recolectaron aproximadamente $180 \mathrm{~g}$ de concentrado, que se molieron, a través de un molino Udy ${ }^{\circledR}$, con criba de $1 \mathrm{~mm}$ y, posteriormente, se analizaron en el laboratorio.

Análisis químicos. A los forrajes y a los concentrados, se les determinó la concentración de materia seca (MS) y cenizas por gravimetría, por medio de los métodos AOAC-930.15 y AOAC-9942.05, respectivamente (AOAC, 2010); proteína cruda (PC), por el método Kjeldahl (Thiex et al. 2002); extracto etéreo (EE), por el método Soxhlet (AOAC, 2010); fibra detergente neutro (FDN) (Van Soest et al. 1991); la energía bruta, se determinó con bomba calorimétrica de chaqueta seca (CAL2k®) y los carbohidratos no fibrosos (CNF), se estimaron por la diferencia $\mathrm{CNF}=\mathrm{MO}-\mathrm{EE}-\mathrm{PC}$.

Conversión alimenticia. Se determinó el índice de conversión alimenticia (ICA), con la fórmula ICA = C / GP, donde ICA es el índice de conversión alimenticia, C y PG son el consumo de alimento ( $g$ ) y la ganancia de peso (g) durante la fase de ceba, respectivamente.

Análisis estadístico. Las variables asociadas a la edad de los animales fueron analizadas bajo un diseño experimental Medidas Repetidas en el Tiempo y utilizando, como factor de bloqueo, el sexo 2x4x5 (Kaps \& Lamberson, 2004), de acuerdo con el modelo: $y_{i j k l}=\mu+\beta_{i}+\tau_{j}+\delta_{j k}+\lambda_{l}+(\tau * \lambda)_{j l}$ $+\varepsilon_{i j k l}$, donde $y_{i j k l}$ es el valor de la observación, $\mu$ es el promedio de la población, $\beta_{i}$ es el efecto del bloque, $\tau_{j}$ es el efecto del tratamiento, $(\tau * \lambda)_{j l}$ es el efecto de la interacción entre el tratamiento y el periodo, $\delta_{j k}$ es el error aleatorio con media
$0 \mathrm{y} \sigma^{2}$, la varianza entre los animales dentro del tratamiento y esta es igual a la covarianza entre las medidas repetidas dentro de los animales y $\varepsilon_{i j k l}$, el error experimental.

Las variables relacionadas con el sacrificio de los animales y la eficiencia de la dieta fueron analizadas bajo un diseño experimental Bloques Completamente al Azar Generalizado utilizando, como factor de bloqueo, el sexo 2x4 (Kaps \& Lamberson, 2004), de acuerdo con el modelo: $y_{i j k}=\mu+\beta_{i}$ $+\tau_{j}+\varepsilon_{i j k}$, donde $y_{i j k}$ es el valor de la observación, $\mu$ es el promedio de la población, $\beta i$ es el efecto del bloque, $\tau_{j}$ es el efecto del tratamiento, $\varepsilon_{i j k}$ es el error experimental.

Los supuestos fueron probados con la metodología indicada para cada caso. Cuando el modelo resultó estadísticamente significativo, se procedió con un análisis de comparación de medias, aplicando la prueba Tukey y cuando fue necesario, se determinó el efecto lineal o cuadrático asociado a la edad. Para el análisis de los resultados, se dispuso del programa SAS versión 9.0 (Copyright(C) 2002 by SAS Institute Inc., Cary, NC, USA).

\section{RESULTADOS Y DISCUSIÓN}

No se presentó interacción entre los tratamientos y la edad de los conejos ( $\mathrm{P}>0.05)$. El suministro de forraje no afectó el peso de los conejos ( $\mathrm{P}>0.05$; Tabla 2 ). Fomunyam \& Ndoping (2000) y Nieves et al. (2009a) indican que los niveles recomendados de $\mathrm{PC}$ en la dieta de conejos de engorde son 200 y $227 \mathrm{~g} / \mathrm{kg}$ de MS, respectivamente. Nieves et al. (2009a) reportan que el nivel recomendado de FDN en la dieta de conejos de engorde es $340 \mathrm{~g} / \mathrm{kg}$ de $M S$. De tal manera, el contenido de PC y FDN del concentrado utilizado en este experimento fue inferior lo recomendado por Fomunyam \& Ndoping (2000) y Nieves et al. (2009a). Nieves et al. (2009a) recomiendan que el contenido de EB del concentrado para conejos de engorde es $3922 \mathrm{kcal} / \mathrm{kg}$ MS, valor que es inferior a la EB del concentrado empleado en este experimento. La baja concentración de PC y FDN del concentrado, sumado su elevado contenido de EB, sugiere que si adicional al concentrado utilizado en este trabajo se suministran forrajes a conejos de engorde, se podría mejorar el balance nutricional de la dieta y optimizar la ganancia de peso de los animales, pues los forrajes son una fuente importante de proteína y de fibra, pero su nivel de energía es bajo; sin embargo, en el presente experimento, el peso de los conejos no se vio afectado por el suministro de los forrajes (Tabla 2).

El peso, la ganancia de peso acumulada y la ganancia de peso relativa ( $g$ de peso obtenido por $g$ de peso al iniciar el experimento) incrementaron de manera lineal con la edad $(\mathrm{P}<0.001)$. Las ganancias de peso diaria y semanal, así como la ganancia de peso relativa durante la semana ( $g$ de peso obtenido por $g$ de peso al iniciar la semana), disminu- 
Tabla 2. Efecto sobre el peso de conejos en fase de ceba, al suministrar T. diversifolia, A. pintoi y T. gigantea.

\begin{tabular}{|c|c|c|c|c|c|c|c|c|c|c|c|c|c|c|}
\hline \multirow{2}{*}{ Ítem } & \multicolumn{4}{|c|}{ Tratamiento $^{1}$} & \multicolumn{5}{|c|}{ Edad, d } & \multicolumn{5}{|c|}{$\mathrm{P}^{2}$} \\
\hline & C & TD & AP & TG & 37 & 44 & 51 & 58 & 65 & $\mathrm{~T}$ & $\mathrm{E}$ & TxE & $\mathrm{L}$ & C \\
\hline Peso, $g$ & 1583 & 1634 & 1558 & 1565 & $976^{\mathrm{e}}$ & $1318^{d}$ & $1600^{c}$ & $1909^{b}$ & $2120^{\mathrm{a}}$ & NS & $* * *$ & NS & $* * *$ & $* *$ \\
\hline \multicolumn{15}{|c|}{ Ganancia de peso, g } \\
\hline Día, g & 43 & 47 & 44 & 42 & $56^{\mathrm{a}}$ & $49^{b}$ & $40^{c}$ & $44^{\mathrm{bc}}$ & $30^{\mathrm{d}}$ & NS & $* * *$ & NS & $* * *$ & NS \\
\hline Semana, $g$ & 304 & 330 & 306 & 291 & $393^{a}$ & $343^{b}$ & $282^{c}$ & $309^{b c}$ & $212^{d}$ & NS & $* * *$ & NS & $* * *$ & NS \\
\hline Acumulada, $g$ & 994 & 1052 & 984 & 976 & $393^{e}$ & $736^{d}$ & $1017^{c}$ & $1325^{\mathrm{b}}$ & $1537^{\mathrm{a}}$ & NS & $* * *$ & NS & $* * *$ & $* *$ \\
\hline \multicolumn{15}{|c|}{ Ganancia relativa $^{3}$} \\
\hline Semana & 0.31 & 0.32 & 0.31 & 0.30 & $0.68^{\mathrm{a}}$ & $0.35^{\mathrm{b}}$ & $0.21^{c}$ & $0.19^{c}$ & $0.11^{d}$ & NS & $* * *$ & NS & $* * *$ & $* * *$ \\
\hline Acumulada & 1.71 & 1.81 & 1.74 & 1.67 & $0.68^{\mathrm{e}}$ & $1.27^{\mathrm{d}}$ & $1.76^{\mathrm{c}}$ & $2.29^{b}$ & $2.66^{\mathrm{a}}$ & NS & $* * *$ & NS & $* * *$ & $* *$ \\
\hline
\end{tabular}

${ }^{1}$ Conejos que recibieron sólo concentrado durante la fase de ceba $(\mathrm{C})$ y conejos que recibieron concentrado más $180 \mathrm{~g} / \mathrm{d}$ de $T$. diversifolia (TD), A. pintoi (AP) o T. gigantea (TG). ${ }^{2} \mathrm{~T}$ : efecto del tratamiento; E: efecto de la edad; TxE: efecto de la interacción entre el tratamiento y la edad; L y C efecto lineal y cuadrático de la edad, respectivamente; NS es no significativo; superíndices con letras diferentes en la misma fila indican diferencias: * es $\mathrm{p}<0,05 ; * *$ es $\mathrm{p}<0,01 \mathrm{y} * * *$ es $\mathrm{p}<0,001{ }^{3} \mathrm{~g}$ de peso obtenido por $\mathrm{g}$ de peso al iniciar la semana y el experimento para las ganancias relativas por semana y acumulada, respectivamente.

yeron de manera lineal con la edad $(\mathrm{P}<0.001)$. Estos resultados indican que el peso de los conejos aumentó hasta los 65 días, pero la velocidad de crecimiento fue menor al avanzar la edad. Las ganancias diarias de peso fueron similares a lo reportado por Fernández-Carmona et al. (1998) y Martínez et al. (2005), quienes reportan ganancias diarias de 37,2-40,3 y 34-46g, respectivamente. Fernández-Carmona et al. (1998) utilizaron concentrados con diferentes niveles de inclusión de alfalfa, mientras que Martínez et al. (2005) dispusieron de concentrados con alfalfa o morera. De igual manera, en la revisión de Maertens (1999), se reportan ganancias de peso diarias (37,9-48), similares a las de este trabajo. En otros trabajos, en los cuales también se utilizaron conejos Nueva Zelanda Blanco, se reportan ganancias diarias de 21,9-29,5g (Fomunyam \& Ndoping, 2000), 22-30g (Fotso et al. 2000) y 25-37g (Nieves et al. 2009b), que son inferiores a lo hallado en el presente trabajo. Fomunyam \& Ndoping (2000) utilizó alimento con diferente tamaño de pellets; Fotso et al. (2000), concentrado con hojas de yuca y, Nieves et al. (2009b), concentrado con diferentes niveles de inclusión de leucaena, quiebrabarrigo o morera. La literatura indica que las diferencias en la ganancia de peso pueden ser explicadas por el potencial genético de los conejos (Maertens, 1999; Ponce de León et al. 2002), la calidad del alimento, las condiciones ambientales (Iyegue-Erakpotobor \& Muhammad, 2008) y el proceso de fabricación del alimento (Fotso et al. 2000).

El suministro de forraje no afectó el consumo de concentrado $(P>0.05)$, pero sí el consumo total de alimento $(P<0.001)$ y el ICA $(\mathrm{P}<0.001)$ (Tabla 3$)$. El mayor consumo de alimento, se presentó en el tratamiento AP y, el menor, para el control; el tratamiento TG no fue diferente frente al control. El ICA fue menor para el tratamiento control, que se puede explicar por la ausencia de efecto del suministro de forraje sobre el consumo de concentrado y el peso de los conejos, así como por el consumo adicional de forraje. El ICA del tratamiento C fue similar a lo encontrado por Maertens (1999), Fotso et al. (2000) y Martínez et al. (2005), quienes reportan 2,88, 2,95 y 3,01 , respectivamente; pero inferior a lo reportado por Onifade \& Tewe (1993), Fomunyam \& Ndoping (2000) y Nieves et al. (2009b), de 4,52-5,51, 4,0-4,5 y 4,2, respectivamente. Nieves et al. (2009b) utilizaron T. gigantea en la fabricación alimento peletizado y reportan que el ICA estuvo entre 6,40 y 6,47 , similar lo encontrado en el tratamiento TG, a pesar que el forraje se suministró de manera fresca.

El suministro de forraje afectó el consumo de MS (P<0.05), que fue mayor para AP frente a los demás tratamientos, excepto en comparación con el tratamiento TD. El suministro de forraje disminuye el consumo de alimento (Martínez et al. 2005; lyegue-erakpotobor et al. 2006), lo cual, no fue el caso para este experimento. Trabajos en los que se incluyen forrajes en el alimento peletizado indican que la inclusión de $30 \%$ de forrajes en la mezcla alimenticia no afecta el crecimiento, el consumo de alimento y la conversión alimenticia (Nieves et al. 2009b) y, en el presente trabajo, el forraje representó el $5,20,5$ y $4.8 \%$ del total de MS consumida, para los tratamientos TD, AP y TG, respectivamente. El menor consumo de alimento en el TG, en comparación con el AP, podría ser explicado por diferencias en la composición de estos forrajes. Iyegue-Erakpotobor \& Muhammad (2008) evaluaron la 
Tabla 3. Efecto sobre el consumo de alimento, de las fracciones del alimento y el índice de conversión alimenticia de conejos en fase de ceba, al suministrar T. diversifolia, A. pintoi y T. gigantea.

\begin{tabular}{|c|c|c|c|c|c|c|c|}
\hline \multirow{2}{*}{ Ítem } & \multicolumn{4}{|c|}{ Tratamiento $^{1}$} & \multirow{2}{*}{$\mathrm{ESM}^{2}$} & \multirow{2}{*}{$\mathrm{CV}^{3}$} & \multirow{2}{*}{$\mathrm{P}^{4}$} \\
\hline & C & TD & AP & TG & & & \\
\hline \multicolumn{8}{|l|}{ Consumo alimento, $\mathrm{g}$} \\
\hline Concentrado & 2759 & 2761 & 2728 & 2537 & 276 & 10,3 & NS \\
\hline Concentrado+Forraje & $2759^{c}$ & $3854^{\mathrm{b}}$ & $4980^{a}$ & $3406^{\mathrm{bc}}$ & 325 & 8,7 & $* * *$ \\
\hline $\mathrm{ICA}^{5}$ & $2,76^{\mathrm{b}}$ & $6,74^{\mathrm{a}}$ & $7,60^{\mathrm{a}}$ & $6,43^{\mathrm{a}}$ & 1,0 & 17,1 & $* * *$ \\
\hline \multicolumn{8}{|l|}{$\begin{array}{l}\text { Consumo fracciones } \\
\text { del alimento }\end{array}$} \\
\hline Materia seca, $\mathrm{g}$ & $2510^{b}$ & $2645^{\mathrm{ab}}$ & $3127^{\mathrm{a}}$ & $2426^{b}$ & 253 & 9,5 & $*$ \\
\hline Proteína cruda, g & $477^{\mathrm{b}}$ & $517^{\mathrm{ab}}$ & $603^{a}$ & $456^{b}$ & 48 & 9,3 & $* *$ \\
\hline Extracto etéreo, g & $105^{\mathrm{b}}$ & $106^{\mathrm{b}}$ & $147^{\mathrm{a}}$ & $109^{b}$ & 12 & 10,1 & $* * *$ \\
\hline $\mathrm{CNF}^{6}, \mathrm{~g}$ & 1102 & 1125 & 1164 & 1035 & 108 & 10 & NS \\
\hline FDN, $g$ & $640^{\mathrm{b}}$ & $684^{\mathrm{b}}$ & $970^{\mathrm{a}}$ & $635^{\mathrm{b}}$ & 65 & 8,9 & $* * *$ \\
\hline FDA, $g$ & $350^{\mathrm{b}}$ & $382^{b}$ & $588^{a}$ & $354^{\mathrm{b}}$ & 36 & 8,5 & $* * *$ \\
\hline Lignina, $g$ & $128^{b}$ & $140^{b}$ & $205^{a}$ & $130^{b}$ & 13 & 8,8 & $* * *$ \\
\hline Cenizas, g & $198^{b}$ & $221^{\mathrm{ab}}$ & $253^{a}$ & $207^{b}$ & 21 & 9,5 & $*$ \\
\hline $\begin{array}{l}\text { Energía bruta, } \\
\text { kilocalorías }\end{array}$ & $109^{b}$ & $114^{\mathrm{ab}}$ & $134^{\mathrm{a}}$ & $105^{b}$ & 11 & 9,6 & $*$ \\
\hline
\end{tabular}

${ }^{1}$ Conejos que recibieron sólo concentrado durante la fase de ceba (C) y conejos que recibieron concentrado más $180 \mathrm{~g} / \mathrm{d}$ de $T$. diversifolia (TD), A. pintoi (AP) o T. gigantea (TG). ${ }^{2}$ Error estándar de la media. ${ }^{3}$ Coeficiente de variación. ${ }^{4} \mathrm{NS}$ es no significativo; superíndices con letras diferentes en la misma fila indican diferencias: * es $\mathrm{p}<0,05$; ** es $\mathrm{p}<0,01$ y *** es $\mathrm{p}<0,001$. ${ }^{5}$ Índice de conversión alimenticia, $g$ de alimento consumido por cada $g$ de peso ganado durante la fase de ceba. ${ }^{6}$ Carbohidratos no fibrosos $=100-$ FDN - PC - EE - cenizas.

aceptabilidad de diversos forrajes por parte de conejos que consumen concentrado y encontraron que dicha aceptabilidad varía según el tipo de forraje, lo cual, afecta el consumo voluntario del forraje.

El consumo de EE, FDN, FDA y Lignina fue mayor para AP, en comparación con los demás tratamientos $(\mathrm{P}<0.001)$. El consumo de $P C(P<0.01)$, cenizas $(P<0.05)$ y energía bruta $(\mathrm{P}<0.05)$ fue mayor para AP, en comparación con los tratamientos control y TG, pero no frente a TD. El suministro de forrajes no afectó el consumo de CNF $(\mathrm{P}<0.05)$. La ausencia de efecto del tratamiento AP sobre el peso, a pesar del mayor consumo de nutrientes, podría ser explicada por una menor eficiencia de la dieta o por el potencial genético de los conejos.

El peso total al sacrificio y el de las diferentes partes del cuerpo no se afectó por los tratamientos (Tabla 4). El suministro de forraje sugiere un aumento en el tamaño del tracto digestivo, que no fue el caso para este experimento, pues no se afectó el peso del intestino, ni de las vísceras. FernándezCarmona et al. (1998) registraron un incremento en el peso del tracto gastrointestinal y del contenido del ciego, debido al incremento de fibra en la dieta. No se evalúo el tamaño, ni el peso del ciego en el presente trabajo; de tal manera, que sería importante determinar, en futuras investigaciones, el efecto de estos forrajes sobre el tamaño del ciego, pues es probable que se hayan presentado diferencias en el peso del contenido del mismo y que dichas diferencias hayan quedado enmascaradas por el peso total de los intestinos.

El contenido de PC del T. diversifolia fue superior a lo indicado por Nieves et al. (2011) y Castaño (2012), quienes reportan concentraciones de 185,2 y $242 \mathrm{~g} / \mathrm{kg}$ de $\mathrm{MS}$, respectivamente; mientras que la concentración de FDN fue similar a 329,4g/kg de MS, reportada por Nieves et al. (2011), pero inferior a $542 \mathrm{~g} / \mathrm{kg}$ de MS, obtenida por Castaño (2012). Los contenidos de PC y FDN del A. pintoi fueron similares a lo indicado por Nieves et al. (2008), quienes reportan concentraciones de 208,0 y $510,7 \mathrm{~g} / \mathrm{kg}$ de $\mathrm{MS}$, para la PC y la FDN, respectivamente. Los contenidos de PC y FDN del $T$. gigantea fueron inferiores a lo reportado por Nieves et al. (2008) y Castaño (2012), quienes indicaron, para la PC, concentraciones de 181,0 y $210 \mathrm{~g} / \mathrm{kg}$ de $\mathrm{MS}$, respectivamente $\mathrm{y}$, 
Tabla 4. Efecto sobre el peso de conejos en fase de ceba, al suministrar T. diversifolia, A. pintoi y T. gigantea.

\begin{tabular}{|c|c|c|c|c|c|c|c|}
\hline \multirow{2}{*}{ Ítem } & \multicolumn{4}{|c|}{ Tipo de dieta ${ }^{1}$} & \multirow{2}{*}{$\mathrm{ESM}^{2}$} & \multirow{2}{*}{$\mathrm{CV}^{3}$} & \multirow{2}{*}{$P^{4}$} \\
\hline & C & TD & AP & TG & & & \\
\hline \multicolumn{8}{|l|}{ Peso, $g$} \\
\hline Animal & 2106 & 2233 & 2103 & 2041 & 137 & 6,5 & NS \\
\hline Canal & 987 & 946 & 1050 & 960 & 117 & 11,9 & NS \\
\hline Piel & 179 & 187 & 209 & 188 & 24 & 12,7 & NS \\
\hline Cabeza & 203 & 205 & 228 & 198 & 29 & 13,8 & NS \\
\hline Patas & 66 & 49 & 63 & 60 & 15 & 25,3 & NS \\
\hline Vísceras & 479 & 457 & 453 & 463 & 32 & 6,9 & NS \\
\hline Intestino & 242 & 228 & 259 & 244 & 30 & 12,3 & NS \\
\hline Corazón & 8,8 & 7,3 & 9,3 & 7,5 & 1,2 & 14,6 & NS \\
\hline Hígado & 51 & 54 & 54 & 52 & 5 & 9,0 & NS \\
\hline Pulmones & 14 & 13 & 12 & 12 & 5 & 36,5 & NS \\
\hline \multicolumn{8}{|l|}{ Pesos relativo $^{5}$} \\
\hline Canal & 46,9 & 42,6 & 49,9 & 47,0 & 6,0 & 12,9 & NS \\
\hline Piel & 8,5 & 8,4 & 9,9 & 9,2 & 1,3 & 14,2 & NS \\
\hline Cabeza & 9,6 & 9,2 & 10,8 & 9,7 & 1,5 & 14,9 & NS \\
\hline Patas & 3,1 & 2,2 & 3,0 & 2,9 & 0,7 & 23,4 & NS \\
\hline Vísceras & 22,8 & 21,0 & 21,6 & 22,7 & 1,5 & 6,6 & NS \\
\hline Intestino & 11,5 & 10,3 & 12,3 & 11,9 & 1,5 & 12,8 & NS \\
\hline Corazón & 0,4 & 0,3 & 0,4 & 0,4 & 0,1 & 15,2 & NS \\
\hline Hígado & 2,4 & 2,5 & 2,6 & 2,5 & 0,2 & 9,9 & NS \\
\hline Pulmones & 0,6 & 0,6 & 0,6 & 0,6 & 0,2 & 36,3 & NS \\
\hline
\end{tabular}

${ }^{1}$ Conejos que recibieron sólo concentrado durante la fase de ceba (C) y conejos que recibieron concentrado más $180 \mathrm{~g} / \mathrm{d}$ de $T$. diversifolia (TD), A. pintoi (AP) o T. gigantea (TG). ${ }^{2}$ Error estándar de la media. ${ }^{3}$ Coeficiente de variación. ${ }^{4} \mathrm{NS}$ es no significativo; superíndices con letras diferentes en la misma fila indican diferencias: * es $\mathrm{p}<0,05, * *$ es $\mathrm{p}<0,01 \mathrm{y} * * *$ es $\mathrm{p}<0,001{ }^{5} \mathrm{~g}$ de peso de la parte del cuerpo por $100 \mathrm{~g}$ de peso del animal al sacrificio.

para la FDN, 450,4 y 492g/kg de MS, respectivamente. Estas diferencias podrían estar relacionadas con las características del suelo, factores medioambientales y la edad de corte.

Es importante evaluar el efecto suministrar diferentes presentaciones, ya sea harina, ensilaje o forraje fresco picado, sobre el desempeño productivo y la eficiencia de la dieta, ya que según Fomunyam \& Ndoping (2000), la forma del alimento afecta el consumo y la utilización del mismo. Un incremento en la proporción de partículas pequeñas incrementa el tiempo de retención en el ciego y la digestibilidad de la fibra, pero disminuye el consumo de MS (García et al. 1999). El colon separa las partículas de acuerdo a su tamaño; las partículas menos densas se mueven rápidamente, a través del colon, gracias a la peristalsis, mientras que los flui- dos y las partículas pequeñas, se desplazan hacia el ciego, debido a movimientos anti-peristálticos, acompañados de contracciones de la haustra (Cheeke, 1986).

La producción de conejos ha cobrado gran interés, debido a que convierten en material fibroso en carne magra (Deshmukh et al. 1993), benéfica para la salud del hombre (lyegue-Erakpotobor, 2007) y es posible alimentarlos con dietas que contienen elevadas proporciones de forrajes (Nieves et al. 2008); sin embargo, los resultados de este experimento indican que el suministro de forraje fresco de T. diversifolia, T. gigantea y A. pintoi no afectó el peso ni la eficiencia de la dieta ni la canal $(P>0.05)$. El uso de forrajes en la alimentación de conejos afecta el proceso digestivo, aprovechamiento de nutrientes y la eficiencia biológica, debido a 
cambios en la tasa de pasaje (Nieves et al. 2011) o velocidad de tránsito. Se ha demostrado un bajo ritmo de crecimiento en los conejos e incremento en los costos de mano de obra, cuando los forrajes no son mezclados como dieta granulada balanceada (Nieves et al. 2009b), ni el alimento ha sido peletizado (Fomunyam \& Ndoping, 2000), de tal manera que para incrementar el uso de los forrajes es necesario su inclusión en dietas balanceadas granuladas (Nieves et al. 2008).

En conclusión, el suministrar forraje fresco de T. diversifolia, $T$. gigantea y A. pintoi en la alimentación de conejos de la raza Nueva Zelanda Blanco, durante la fase de engorde, no afecta el peso, ni el consumo de concentrado, ni la calidad de la canal.

Agradecimientos. Al Laboratorio de Nutrición Animal de la Facultad de Ciencias Pecuarias de UNISARC, por el apoyo en el análisis químico. Financiación: Este trabajo se realizó con recursos del Ministerio de Agricultura y Desarrollo Rural de Colombia. Conflicto de intereses: El manuscrito fue preparado y revisado por los autores, quienes declaramos que no existe conflicto de intereses, que ponga en riesgo la validez de los resultados presentados.

\section{BIBLIOGRAFÍA}

1. AOAC. 2010. Official methods of analysis of AOAC International. AOAC Intenational (Maryland). 77p.

2. CASTAÑO, G.A. 2012. Efecto del proceso del ensilaje sobre el valor nutricional de Pennisetum purpureum, Tithonia diversifolia y Trichanthera gigantea. Inv. Unisarc (Colombia). 10(2):22-36.

3. CHEEKE, P.R. 1986. Potentials of rabbit production in tropical and subtropical agricultural systems. J. Anim. Sci. (United States). 63(5):1581-1586.

4. DESHMUKH, S.V.; PATHAK, N.N.; TAKALIKAR, D.A.; DIGRASKAR, S.U. 1993. Nutritional effect of mulberry (Morus alba) leaves as sole ration of adult rabbits. World Rab. Sci. (España). 1(2):67-69.

5. FERNÁNDEZ-CARMONA, J.; BERNAT, F.; CERVERA, C.; PACUAL, J.J. 1998. High lucerne diets for growing rabbits. World Rab. Sci. 6(2):237-240.

6. FOMUNYAM, R.T.; NDOPING, B.N. 2000. Utilization of pelleted and no pelleted feed by growing rabbits in tropical conditions. World Rab. Sci. 8(2):61-62.

7. FOTSO, J.M.; FOMUNYAM, R.T.; NDOPING, B.N. 2000. Protein and energy sources for rabbit diets in cameroon: 1-Protein sources. World Rab. Sci. 8(2):57-60.
8. GARCÍA, J.; CARABAÑO, R.; DE BLAS, J.C. 1999. Effect of fiber source on cell wall digestibility and rate of passage in rabbits. J. Anim. Sci. 77(4):898-905.

9. IYEGUE-ERAKPOTOBOR, G.T.; ALIYU, R.; UGURU, J. 2006. Evaluation of concentrate, grass and legume combinations on performance and nutrient digestibility of grower rabbits under tropical conditions. Afr. J. Biotechnol. (South Africa). 4(20):2004-2008.

10. IYEGUE-ERAKPOTOBOR, G.T. 2007. Effect of concentrate and forage type on performance and digestibility of growing rabbits under sub-humid tropical conditions. Asian J. Anim. Vet. Adv. (United States). 2(3):125-132.

11. IYEGUE-ERAKPOTOBOR, G.T.; MUHAMMAD, I.R. 2008. Intake of tropical grass, legume and legume-grass mixtures by rabbits. Tropical Grasslands (Australia). 42(2):112-119.

12. KAPS, M.; LAMBERSON, W.R. 2004. Biostatistics for animal science. Cabi Pubishing (London). 445 p.

13. MAERTENS, L. 1999. Towards reduced feeding costs, dietary safety and minimal mineral excretion in rabbits: a review. World Rab. Sci. 7(2):65-74.

14. MARTÍNEZ, M.; MOTTA, W.; CERVERA, C.; PLA, M. 2005. Feeding mulberry leaves to fattening rabbits: effects on growth, carcass characteristics and meat quality. Anim. Sci. (United Kindom). 80(3):275-281.

15. NIEVES, D.; MONCADA, I.; TERÁN, O.; GONZÁLEZ, C.; SILVA, L.; LYKOS, T. 2009a. Parámetros digestivos en conejos de engorde alimentados con dietas basadas en forrajes tropicales: digestibilidad ileal. Bioagro (Venezuela). 21(1):33-40.

16. NIEVES, D.; SCHARGEL, I.; TERÁN, O.; GONZÁLEZ, C.; SILVA, L.; LY, J. 2008. Estudios de procesos digestivos en conejos de engorde alimentados con dietas basadas en follajes tropicales: digestibilidad fecal. Rev. Cient. (Méjico). 18(3):271-277.

17. NIEVES, D.; TERÁN, O.; CRUZ, L.; MENA, M.; GUTIÉRREZ, F.; LY, J. 2011. Digestibilidad de nutrientes en follaje de arnica (Tithonia diversifolia) en conejos de engorde. Trop. Subtrop. Agroecosyst. (Méjico). 14(1):309-314.

18. NIEVES, D.; TERAN, O.; VIVAS, M.; ARCINIEGAS, G.; GONZÁLEZ, C.; LY, J. 2009b. Comportamiento 
productivo de conejos alimentados con dietas basadas en follajes tropicales. Rev.Cient. (Venezuela). 19(2):173-180.

19. ONIFADE, A.A.; TEWE, O.O. 1993. Alternative tropical energy feed resources in rabbit diets: growth performance, diet's digestibility and blood conposition. World Rab. Sci. 1(1):17-24.

20. PONCE DE LEÓN, R.; GUZMÁN, G.; QUESADA, M.E. 2002. Crecimiento y eficiencia alimentaria de cuatro razas de conejos. Rev. Cubana Cienc. Agri. 36(1):714.

21. THIEX, N.J.; MANSON, H.; ANDERSON, S.; PETACCHI, F. 2002. Determination of crude protein in animal feed, forage, grain, and oilseeds by using block digestion with a copper catalyst and steam distillation into boric acid: collaborative study. J. AOAC Int. (United States). 85(2):309-317.

22. VAN SOEST, P.J.; ROBERTSON, J.B.; LEWIS, B.A. 1991. Methods for dietary fiber, neutral detergent fiber, and nonstarch polysaccharides in relation to animal nutrition. J. Dairy Sci. (United States). 74(10):3583-3597.

Recibido: Julio 23 de 2014

Aceptado: Abril 14 de 2015

\section{Cómo citar:}

Castaño, G.; Cardona, J. 2015. Engorde de conejos alimentados con Tithonia diversifolia, Trichanthera gigantea y Arachis pintoi. Rev. U.D.C.A Act. \& Div. Cient. 18(1): 147-154. 